C2020. Licensed under the Creative Commons Attribution-NonCommercial-NoDerivatives 4.0 International http://creativecommons.org/about/downloads

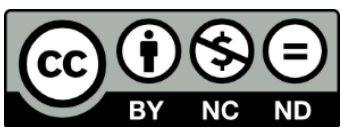

This is the accepted version of this item. The version of record is available at https://doi.org/10.1016/j.newideapsych.2020.100812 


\title{
Mapping Systemic Resources in Problem Solving
}

\author{
Frédéric Vallée-Tourangeau and Gaëlle Vallée-Tourangeau \\ Kingston University
}

\begin{abstract}
Author Note
Correspondence for this article can be addressed to Frédéric Vallée-Tourangeau, Department of Psychology, Kingston University, Kingston-upon-Thames UNITED KINGDOM KT1 2EE, f.vallee-tourangeau@kingston.ac.uk or Gaëlle Vallée-Tourangeau, Department of Management, Kingston University, Kingston-upon-Thames, UNITED KINGDOM, KT2 7LB g.vallee-tourangeau@kingston.ac.uk.. We thank Wendy Ross for helpful discussions that helped us shape some of the arguments presented here, as well as two anonymous reviewers for their comments on a previous version of this manuscript.
\end{abstract}




\begin{abstract}
In the wild, thinking demonstrably uses interactive processes that draw on a wide range of external resources, spanning multiple time scales. As Malafouris (2015, p. 361) puts it, "cognition is not a within property; it is an in-between process". Interactive processes configure extended systems within which each human agent is embedded. Yet much research on higher cognition, such as problem solving, reflects an implicit but deep commitment to methodological individualism that casts the agent as the ontological locus of cognition, and largely dictates the nature of the research enterprise. Thus, tasks to measure capacities and gauge reasoning performance are designed in a manner that reduces or eliminates the possibility of interacting with the problem presentation; if thinking takes place in the head, there is no need or reason to engineer procedures wherein agents can interact with the task's physical constituents. Conversely, a methodological interactivism forces one to acknowledge the participative yet not all-encompassing role of capacities such as working memory and thinking dispositions; it also encourages the granular mapping of the cognitive ecosystem from which new ideas emerge. To adopt an interactivist perspective is thus to focus on the cognitive resources of the extended system inviting a careful description of how these resources are dynamically configured over time and space to promote the development of new ideas in problem solving. In turn, a systemic perspective encourages the development of interventions that promote cognitive performance through the optimisation of systemic rather than individualist cognitive resources.
\end{abstract}




\section{Mapping Systemic Resources in Problem Solving}

We stand in front of a work of art, a painting, a sculpture, we finish an engrossing novel, and marvel at the ingenuity, originality, vision, and craftsmanship of the creative artist. The historicity and complex creative trajectory of the work of art are collapsed in the finished product and encourages us naively to ascribe all these properties to the artist. The short biography of the work or of the artist, that stands solemn alongside, exacerbates this attribution bias: that the work can be explained and understood in terms of properties internal to the artist, his or her ability to think differently and conceive the creative blueprint that resulted in the finished product. The inability (or unwillingness) to investigate the creative arc coupled with a pervasive attribution bias are two important factors that fuel the myth of genius (Weisberg, 1993). To understand the creative arc involves assembling a detailed historical record of the preliminary sketches, the experiments with different materials and techniques and their results, the serendipitous events experienced by the artist in the course of the project, and access to the artist's personal notes and diaries. The fuller the description of the creative arc, the more complete the explanation of its genesis. Interviewing the artist after the work is completed will likely result in a narrative that veils and smooths over the microprocesses that scaffolded its production (March, 2019; Vallée-Tourangeau \& March, 2019).

What participants say or do in a cognitive psychology experiment is rarely a work of art. Still, answers to a few decontextualized questions are traditionally considered diagnostic and inform models of thinking. Psychological researchers separate participants from the complex multiscalar meshwork of resources assembled when people do thinking in the world. This meshwork of resources configures a dynamic and contingent cognitive system. New ideas are emerging from the interactive forces that maintain and dynamically reshape this distributed network of resources; a new research methodology is needed to map this interacting network, to chart how thinking is realised, how new ideas are generated, beyond 
postulating that they are the result of factors such as intelligence or working memory capacity (defined and operationalized in turn by psychometric instruments designed to measure them). Psychologists assess so-called higher-level cognition under controlled conditions. The measuring instruments employed are tasks designed to require the purported ability, capacity, processing, disposition or knowledge necessary to perform rationally, that is produce answers that align with a normative benchmark. The development of these tasks sometime reflect substantial factor analytic scale construction efforts - as is the case for established intelligence tests - and/or theoretically motivated design such as in the development of working memory span tests (e.g., Daneman \& Carpenter, 1980, and their subsequent incarnation, e.g., Engle, Tuholski, Laughlin, \& Conway, 1999). Deductive and inductive reasoning tasks are sometimes designed to cue a prepotent response, not unlike how some insight problems are crafted to encourage a misleading interpretation of the problem that must be overcome to achieve a solution; Wason's $(1960,1966)$ 2-4-6 and selection tasks are good examples, as is Frederick's (2005) cognitive reflection test. The whole heuristics-andbiases research edifice is erected on the basis of such tests (e.g., see the appendix of Toplak et al., 2011, for an illustration of the range of pen and paper based problems that are used in this paradigm).

Interacting with the physical environment — and engaging with the material worldenacts cognitive products that would otherwise not manifest in the absence of interactivity. The Scrabble player moves her letter tiles when generating candidate words (the more they move the tiles, the more words they produce; Ross \& Vallée-Tourangeau, 2020); expert Tetris players rotate the falling zoids more than novices (Kirsh \& Maglio, 1994). Why interact with the world if doing thinking is a pure exercise in offline mental gymnastics (Kirsh, 2010)? Clearly, people can conjure up images of distant places and lost friends, simulate actions and rehearse plans, in the absence of physical prompts. As Hutchins (2010) 
argues, academics are particularly good at this, and similar forms of offline cognition can be elicited from participants in cognitive psychology experiments. But, as Clark (2010, p. 24) is fond of stressing, "it is surely worth noting just how much of our cognitive activity is not like that; brains like ours go to extraordinary length to avoid having to resort to fully environmentally detached reflection" (emphasis in the original). Thinking offline, in turn, involves a great deal of internalised interactivity: "private reflection is a deeply cultural practice that draws on and is enacted in coordination with rich cultural resources" (Hutchins, 2010, p. 702).

Thinking as an activity scaffolded by objects-i.e., the cognitive products of this cognitive ecosystem--invites a granular capture of these objects as they are reified through interactivity. Methodologically, looking at what people do, at what they build, how objects are recruited to support new thoughts, involves a change of focus, a shift in gaze, looking at the world rather than peering at the mind. Yet, we are confronted with cognitive science's insistence that ideas originate in the head, that their origin is best explained through the manipulation of mental representations of the world. There are possibly different strands of cultural, philosophical, ontological and methodological influences that conspire to reinforce and maintain this position. Hutchins's (1995) epilogue to Cognition in the wild plausibly outlines how the computer metaphor of the mind played a part in internalizing the computational functionality of an agent-world interactive system. 21st Century exigencies of psychological science, encouraging the publication of multi-study papers, only possible through the reliance on MTurk workers, Qualtrics and the internet, undoubtedly reinforce this stance (see also Baumeister, Vohs, \& Funder, 2007). There is a folk subjective feel to ideas being in the head too: Personal and scientific narrative smooths over the trial and error toiling in the dark, compressing the past, the history of this trajectory, in neat capsules of insight (see Latour \& Woolgar's [1986] sociological analysis of "thought processes"). There is a 
persistent fundamental attribution error, that feeds internalist thinking; it is also a form of neoliberalism that permeates neuroscientific thinking (Pitts-Taylor, 2010). All these undercurrents blind us to a simpler but fascinating proposal: ideas are constructed, crafted through networks of heterogenous objects, constraints and affordances.

Once an idea is obtained, the circumstances that led to the moment of discovery are forgotten. These circumstances are the real heterogenous microprocesses (serendipitous, possibly, polytemporal certainly) that pave the way to an inference. This construction can be mentally simulated, but it is much harder, and 'real scientists' do not do this, they do not work hard to mentally simulate things, they work hard rather to produce artefacts and physical traces that lend themselves to simple perceptual judgments; they look at things (see Latour's [1999] pedocomparator observations in Pandora's hope).

The predominant research paradigm in the psychology of thinking presents participants with 'reasoning' tasks in impoverished cognitive ecosystems, decoupled from real-world pragmatics, devoid of utilities, and with limited opportunity to scaffold and reify cognitive processes through interacting with a physical problem presentation or exploit its spatial arrangement. Performance on such tasks, as if any other outcomes were surprising, generally exhibits systematic biases and shortcomings, falls prey to illusions and other framing legerdemains, and reflects what seems to be muted rationality and prosaic creativity. Cognitive psychologists labour under the auspices of methodological individualism (ValléeTourangeau \& Vallée-Tourangeau, 2014) and these paradigm blinders orient their attention away from the constitutive role of the broader cognitive ecosystem and sharpen the research focus on the properties of the 'mind'.

Responsibility for the underwhelming performance on these reasoning tasks is apportioned among four types of properties: (i) cognitive capacity; (ii) thinking dispositions; (iii) quality of processing; and (iv) content or knowledge (Toplak et al., 2011). Capacity 
includes factors such as working memory capacity and the efficient retrieval of long-term memory information. Thinking dispositions are attitudes towards effortful thinking and open mindedness. Quality of processing relates to the common finding that "humans [tend to be] cognitive misers because their basic tendency is to default to heuristic processing mechanism of low computational expense" (Toplak et al., 2011, p. 1283). Finally, content variables refer to knowledge schemas in long term memory acquired through relevant experience and applied to solve new problems. Psychometric efforts generally proceed by measuring a participant's in-the-head cognitive capacity, processing and thinking dispositions, and correlating his or her performance on a range of reasoning problems (as described above). Unsurprisingly, these mental capacities and dispositions explain a significant proportion of variance in reasoning performance; yet blunt measures of performance, such as producing normative answers to logical or probabilistic reasoning problems, do not in themselves explicate how thinking happened. Measures of working memory capacity correlate with measures of intelligence (Engle et al., 1999) which correlate with measures of deductive and causal reasoning (Stanovich \& West, 1998) and problem solving (e.g., Gilhooly \& Fioratou, 2009; Davidson, 1995). Thinking is conceived as a product of the mind: one that is better engineered, with larger storage and more efficient processing, simply performs better at these tasks. We would argue that the statistical success of this approach does not entail that it offers a representative characterisation of thinking as observed or enacted outside the laboratory. As a psychometric exercise, this approach is at best paramorphic: It can predict performance, but it says little about behaviour, or about how solutions to problems are actually developed. If interactivity is at the heart of cognition, and we mean this in the strongest ontological sensethinking is "not a within property, it is an in-between process" to adapt Malafouris $(2015, \mathrm{p}$. 361) - then laboratory-based exploration of thinking and reasoning should proceed with tasks engineered to permit and encourage interactivity (see also Bickhard, 2009). More important, 
observing (and filming) participants do thinking in interactive environments help researchers provide a granular description of the reciprocal and co-constitutive changes in the physical environment and in participants, unveiling the microprocesses that scaffold the development of a new thought.

\section{Insight Problem Solving}

Cognitive psychologists working on insight problem solving often preface their work with short popular science vignettes of innovation and discovery (Weisberg, 2018). In their efforts to scale down problem solving in a manner that can be examined under laboratory conditions they focus on certain aspects of this innovation folklore, foremost among them is the alleged suddenness of the discovery after months of toiling in relative darkness, which in turn seems to point at a felicitous conceptual rearrangement in the head of the creative innovator. The cognitivist take on this conceptual transformation is cast in terms of mental representations of the problem, of the world, and the mental processes through which these mental representations changed in a manner that now offer a solution to the vexing problem.

The disciplinary conceptual framework of the cognitive psychologist naturally orients her focus on content and mental processes, away from the broader physical and social context in which discovery happen. Participants are presented with batteries of simple riddles and word associate problems, each presented for a few minutes (or seconds) and participants' performance is aggregated in terms of solution rates. Problems vary in their numerical, logical or visuo-spatial aspects; they are made easier or harder to solve (by adding irrelevant and misleading premises); the phenomenology of discovering the answer is sometimes measured, to capture the qualia of 'aha' moments. Sometimes participants are invited to narrate their efforts to solve these problems, and these verbal protocols are taken as a window onto working memory content and problem-solving strategies. Sometimes participants are 
immobilized in a scanner, and the neural correlates of insightful solutions are imaged in coloured areas of brain activity.

The focus on the individual and the content of his or her mental representations of the world has informed a cognitive science of creativity. These research efforts sought to elucidate the role of working memory and intelligence in creativity, as operationalised in terms of the correlation between these psychometric measures and the solution rates for these simple riddles (Chuderski \& Jastrzębski, 2018); in addition, correlational evidence provides some support for the associations between features of personality, subclinical psychopathology and creative problem solving (e.g., Zabelina, Condon, \& Beeman, 2014). Notable progress in the identification of the putative unconscious processes in incubation (Gilhooly, Georgiou, \& Devery, 2013) should be mentioned as well as the neuroscience efforts that have implicated the right temporal lobe on the phenomenology of insight (Kounios \& Beaman, 2014).

We wish to argue that lab-based problem-solving research should be more attuned to key features of creative problem solving by scientists, artists and engineers (see Glaveanu et al. 2013), and at the same time resist fetishizing the innovator's mind; cognition is a term that is taken to mean mental, yet its etymology relates to knowledge, not where it is store nor how it is obtained (the etymology of intelligence is also interesting and takes us beyond the mental; Latour, 1999; see also Malabou, 2019). The pertinence of the traditional research questions that has animated much of the research on creative problem-solving wanes considerably once the focus on the mind is loosened. In turn, the key feature of creativity resides in working with things, objects, traces. As such, then, there is an inevitable physical interaction with something outside the mind, something in the world. Creativity is realised through interactivity. If thinking proceeds through things, then these things must be examined, and carefully. The actions with these things should too. The reciprocal change in 
the innovator and in the world as a result of these actions should also be an object of study. Actions and changes have spatial and temporal coordinates that chart a trajectory. But the trajectory is not a ballistic one: There is no calculus of mass and velocity that enables precise destination predictions. The trajectory is a contingent one, and as such the charting cannot be done a priori, only a posteriori. The methodological consequences for problem-solving research are profound.

A theoretical framework is erected on ontological assumptions. If one is committed to understanding creativity and problem solving in terms of cognitive abilities and capacities, attention is naturally deflected away from the situated character of cognition. Researchers demonstrate an indifference to--and sometimes ignorance of--how new ideas emerge from the interaction with the world, from the agent-word coupling, and this indifference is reflected in their methodological decisions (which may exacerbate their inability to see how ideas emerge and evolve). A good example of this research strategy is offered in Chuderski, Jastrzębski and Kucwaj (2020) who proclaim to offer a test of the importance of interactivity in insight problem solving. As we illustrate below, the ontological assumptions guide decisions on procedure and measures in a manner that prevents researchers from understanding how new ideas are generated in problem solving.

The authors compare performance on a set of 9 classic insight problems in two groups of participants; one group works on these problems with pen and paper (which, incidentally affords a fair deal of interactivity with external representations) and in the other participants are offered artifacts corresponding to the problem elements and are tasked to build a model of the solution. Participants were run in groups over a five-hour testing session. A battery of working memory tasks preceded the nine insight problems, and the order of the problems was held constant from easier problems to hard problems. The problems were themselves a mix of numerical, logical and visuo-spatial problems (this mix presumably motivated by a 
generalizability concern). Participants were given up to 2 minutes to solve the easy problems, up to 4 minutes for the intermediate problems, and up to 8 minutes to solve the hard problems (performance was at floor for these problems, and after 4 minutes elapsed, participants were given a hint); thus participants completed nine problems in under 45 minutes. Solution rates were very low across all problems and conditions (under $25 \%$ on average), and particularly for the hard problems: Solution rates were marginally higher in the interactivity condition for four problems, higher in the pen and paper condition for another four problems, and at floor for one problem.

Running participants in groups and running a large number of participants in such a manner, makes it hard--although not impossible--to film them such as to provide evidence of the nature and type of actions engaged by participants in the interactive conditions, the type of objects that were created in working out solutions, and to provide a detailed chronometric measure of performance. A more granular analysis of behaviour in either the pen and paper, and more important, in the interactive condition is not offered in this paper. This is a strategic oversight motivated--even validated--by a certain ontological framework. Thus, the data resolution is poor and the fundamental psychological question of how new ideas are produced cannot be answered. A more granular analysis of individual behavior traces, either in the scribbles from the pen and paper condition or in the gestures and manipulations in the interactive conditions, is where the essence of creative thinking lies. If we want to understand the origin of creative cognition, we need to understand how the type and characteristics of idiosyncratic interactions with the world resulted in an insightful solution. From an interactivist perspective, the fundamental question is not whether interacting with the world alone always supports creative performance. This would amount to asking the cognitivist whether working memory and intelligence alone can always explain creativity. Neither question is productive. A more productive approach, we argue, is to ask when and how 
interaction affordances in the world contribute to the creative process, what tools or artefact are fit to support creative cognition, and for whom. These questions do not exclude the importance of internal representations and cognitive abilities for understanding creativity but they highlight the need to go beyond a blunt measure of performance under unrealistic conditions. A systemic perspective invites the careful mapping of how internal and external resources are co-constituted in the process of reifying new ideas.

Data such as those presented in Chuderski et al. (2020) tell us noting about how participants might have interacted with the artefacts or indeed whether they interacted with them at all (see Ross \& Vallée-Tourangeau, 2020): We simply do not know. Aggregate measures of performance (namely solution rates) are resolutely steadfast in their silence on how solutions are derived, what kind of proto models of solutions are constructed (in the interactivity condition), what kind of partial solutions are sketched in the pen and paper condition. The procedure designed by Chuderski et al. for the interactivity condition reflects either a disingenuous or insouciant consideration of the materiality and affordances of the artefacts provided to participants: Chuderski et al. offer no principled arguments as to how or why the interactivity versions of the problems were designed the way they were. In the one opportunity they had to directly replicate a procedure which had been previously employed to illustrate a substantial transformation in performance through interactivity, namely with the 17 animals problem reported in Vallée-Tourangeau et al. (2016), Chuderski et al. chose to modify every aspect of the problem presentation, from the number of animals to, and more important, the material offered to participants. Chuderski et al. then inoculate these unprincipled decisions with a generalizability argument:

"However, because for one problem there was a clear advantage in the interaction condition, it is possible that some specific problems may benefit from physical interaction, especially when static presentation prevents one from recognizing promising possibilities 
(like 3D solutions) and forces invalid interpretations (2D solutions) instead. Thus, previous single-problem studies may indeed have detected the beneficial effect of physical interaction in certain cases, but they failed to provide a general enough view to recognize this effect as being the exception rather than the rule.” (p. 19)

Nature of Creative Thinking. Scientists build things and manipulate objects; they instrumentalize procedures to generate visual data that can be perceptually inspected. Problems are not solved in the head, but rather problem-solving proceeds with and through external representations. Scientists do not work hard to mentally simulate things, they work hard rather to produce artefacts and physical traces that lend themselves to simple perceptual judgments; they look at things (Latour, 1999). Discoveries involve interacting with physical models (e.g., Watson's carboard models of the base pairs; Latour \& Woolgar, 1986; Weisberg, 2006) and often involve accidental juxtapositions of things and events (Copeland, 2018).

Chuderski et al.'s (2020) position on the nature of creative thinking is not explicitly stated in their paper, but it can be inferred from their procedure, measures and analyses (a clearer statement of the theoretical position can be found in Chuderski \& Jastrzębski, 2018). People solve insight problems like they do non-insight problems, that is in a manner similar to solving mental arithmetic problems and other analytic problems (e.g., river crossing problems or the Tower of Hanoi). There's nothing special about the processes involved in insight problem solving; rather a solution is gradually developed on the basis of deliberate effortful analysis of the problem (we too espouse a form of gradualism, and on this point, we share an affinity with Chuderski and Weisberg). Thus, to explain creative ideation, an agent equipped with better cognitive capacity gauged in terms of intelligence and working memory capacity, will be better able to come with new ideas and solutions to problems. Chuderski et al. take a loan on intelligence with no intention of ever paying it back: Creativity is explained 
in terms of intelligence, and that is the end of the explanation. Ideas are mental representations and new ideas are new mental representations of the world. There is a tautology lurking here: the origins of new ideas can be traced to new ideas. Chuderski et al.'s position is a vestigial form of Cartesian dualism: There is a clear demarcation between the mental and the physical, and psychology is about the mind detached from the world. This position is particularly clearly exposed when they write about the role of accident and trial and error in creativity (p. 18):

"In the [interactive] matchstick algebra problem, it is arguably easier to arrive at the solution by accident or trial and error, for instance by realizing as a result of a random movement of a stick that it could act as a negative sign."

This suggests that accidents, random movements and trial and error are theoretically unimportant features of creativity, and in fact should be discounted, sullying as they do a clearer window onto the mental deductive prowess of the reasoner. Yet, these strategies and so-called data-driven restructuring events (changes in the world that change a reasoner's understanding of the problem, see Fleck \& Weisberg, 2013) are common features of creativity and innovation in science, design and the arts. In fact, these features invite us to think of the creativity as emerging from the interactive and transactional coupling between an agent and the world. Rather than dismissing these features as noise, they should be taken as key elements of creativity, which in turn encourages a radically different ontology of cognition in general, and creativity in particular.

\section{Ideas Distilled through Action}

Mobilizing the worldly phenomenon of problem solving requires an interactive problem-solving task and an object that embodies different phases of the trajectory to solution, to the birth of a new idea. This object is a physical model of the solution, at different stages of construction. When the agent is first confronted with this object, the solution is 
invisible. The agent must accept to use the object as a potential model of the solution and exploit its transformation to explore different paths to solution. Changes to the object are observed and prompt new actions. The procedure can be carefully designed to capture the temporal and physical modification of the object. The creative cognition researcher, in turn, can use these data points to create her own inscription device (cf. Latour \& Woolgar, 1986) that illustrates the developmental phases of discovery, a device for her own benefit as a researcher as well as for the benefit of her college of peers, fellow researchers in problem solving and creativity.

We recently developed an interactive version of a classic so-called insight problem, the triangle of coins (see Figure 1a; Vallée-Tourangeau et al., under review). In this problem, 10 coins are arrayed in a triangular shape. The goal is to move three and only three coins to transform the shape from a triangle pointing down to one pointing up; the solution involves moving the three corner coins. We instrumentalized the traditional presentation in the following manner: We labelled each coin (Figure 1b; the solution involves moving coins Q, W and R, colour coded here in red, although the coins were not coloured in this manner for the participants) and we presented the problem on a 9x9 grid with labelled columns and numbered rows (Figure 1c) which would enable us to trace precisely which coins were moved and where over time (capturing the dynamic changes in the problem configuration). We transformed the problem into an interactive game on a computer tablet, and filmed participants in an observation lab with three camera angles as they worked on the problem to discover its solution. While participants were significantly more likely to solve the problem when they could interact with the model of the problem, that is by moving coins on the tablet, the focus of our discussion here is on how the instrumentalization of the procedure enabled the capture of the origin of the solution: we can explain a participant's solution to the problem not in terms of his or her working memory capacity and intelligence, not in terms of 
a putative restructuring of a mental representation, but rather by describing how the physical environment was modified gradually into a model of the solution.

(a)
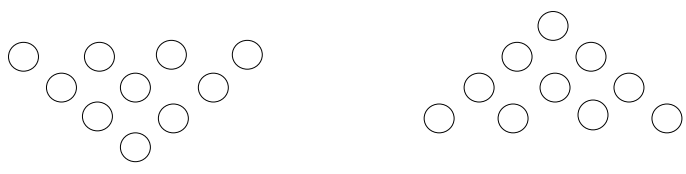

(b)

(a) (S) (L) (I) (T) (I)

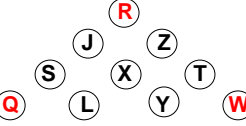

(c)
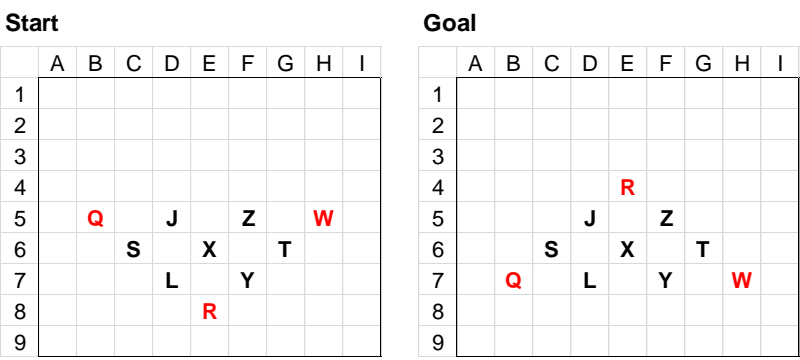

Figure 1. Instrumentalizing the triangle of coins problem.

Our procedure helped us track closely each move made by the participant (as revealed by the position of the coins on the 9x9 grid of coordinates), as well as the latency for each move. The resulting data enabled us to chart the gradual modification of the problem over time and see how the problem was solved. A minority of participants (fewer than 10\%) solve the problem under one minute and sometimes with the minimum number of moves, namely three; most do not. On the basis of the video evidence, we can chart the frame by frame transformation of the problem wrought by individual moves. For example, Figure 2 illustrates the last 16 moves by one of our participants leading to the discovery of the solution (the goal state location of the three corner coins is colour coded here, although it was not for the participants).

This transcription illustrates the discovery of the solution. Once the vertices are identified as promising coins to unlock a solution, they are moved very gradually to their respective, correct, locations on the checkerboard. The solution takes shape slowly. The 
distinction between the agent's knowledge of the solution and the physical realization of the solution is difficult to make, in fact impossible: what the solution is (an ontological question) and what the agent knows (an epistemological one) are inseparable. The agent has discovered the solution once it is constructed, but not before.
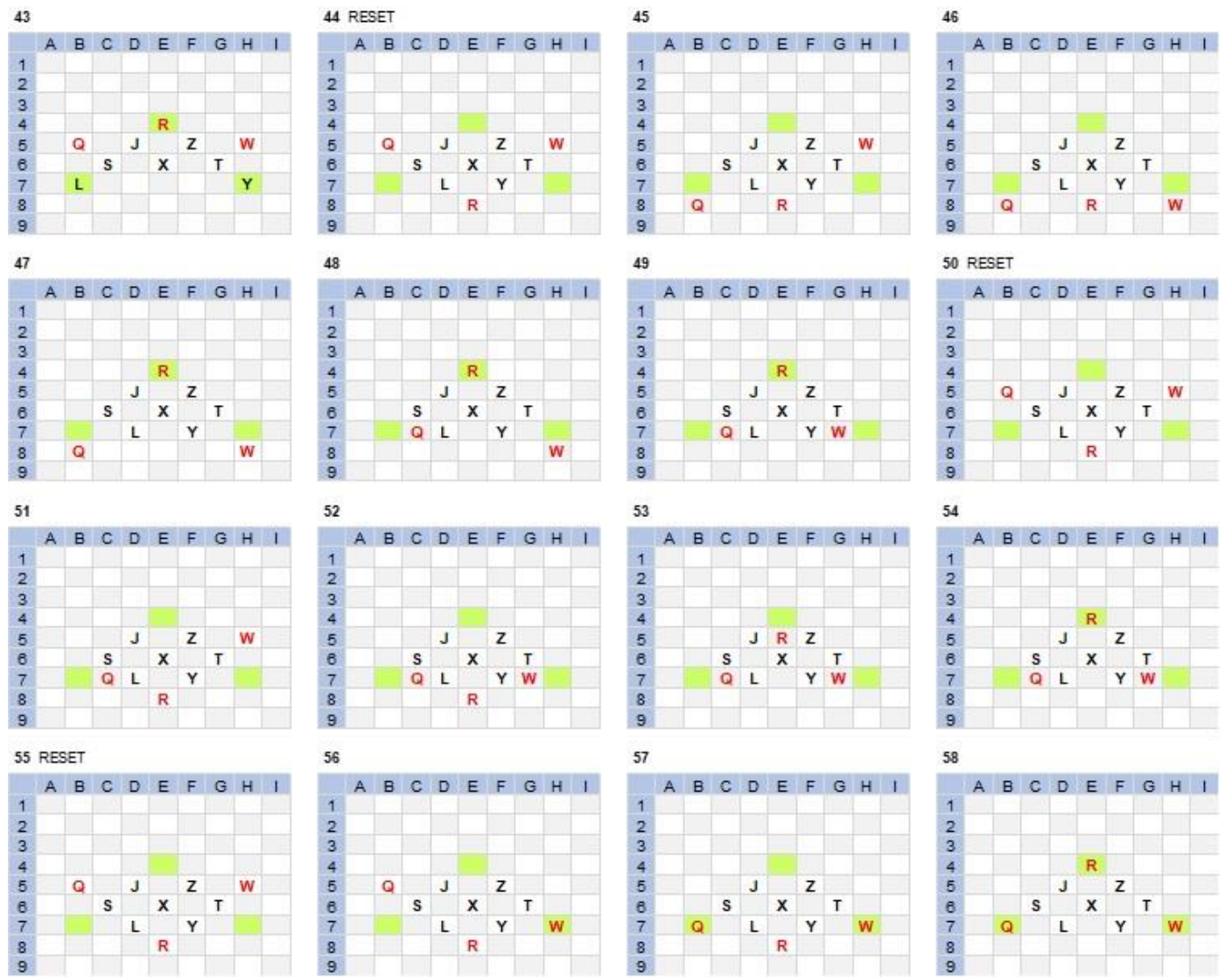

Figure2. Sixteen moves by a participant discovering the solution to the triangle of coins problem (adapted from Vallée-Tourangeau et al. under review).

Interactivity couples an agent to the world and configures a cognitive ecosystem. Cognitive results are produced by this ecosystem (cf. Giere, 2006) and as such interactivity is an ontological substrate (Steffensen, 2013): the process from which new ideas are formed. Cognitive results emerge over time and space and reflect multiscalar tensions. At very short time scales, perception-action loops chart the problem solving trajectory, at slower time scales the reasoner reflects on results, taking stock of the past and formulating hypotheses as 
to what should be done next (or what we termed "moving fast, thinking slow"; ValléeTourangeau \& Vallée-Tourangeau, 2020). Problem solving in the real world unfolds along a spatio-temporal trajectory dotted with boundary objects (Vallée-Tourangeau \& March, 2019; Fiore \& Wiltshire, 2016), that is models of proto-solutions. These boundary objects are transformative and unveil new possibilities for change, cueing new actions that yield new results, creating an iterative action-change cycle that modifies both these boundary objects and the reasoner (as illustrated in Figure 2). Problem solving proceeds along different time scales, and perception-action loops need not be mediated by complex representations. Changes to the world guide new actions. Solutions can be perceived rather than mentally calculated.

Designing an interactive problem solving task environment can reveal the origin of new ideas, that is how the resources available to participants might have been configured to build or sketch a model of the solution, or alternatively and equally important, how these resources were configured in a way that prevented participants from discovering a solution. Designing an interactive problem solving environment transforms thinking, changing it from a second order process to a first order one; in itself this does not mean that it will inevitably scaffold better or more normative performance, although in many cases it can. The more important point is one of methodological granularity: the actions that couple agent to the world and configure as a result a system can be coded, the Promethean nature of the configuration can be analyzed to map the origin of ideas, some more productive than others.

Malafouris (2014) argues that creativity "is not the kind of psychological process that one could quantify by adapting the usual creativity measurements (e.g., divergent thinking tests and insight questionnaires)" and this because the generation of new ideas is a process that is characterised by its dynamic, situated and emergent properties that "defy the psychometric, individualistic, and largely Westernised logic of such measurements, as well as 
their misleading emphasis of an individual genius or talent as the main source of new-idea generation" (p. 148). To start explaining problem solving one must examine the distributed resources of a system configured through the dynamic coupling of the reasoner to her material environment. Insight is attributed not so much to a new way of looking at the problem, but to the new way a problem can look once its physical appearance has been shaped and reshaped through interactivity (and hence the term 'outsight' might be more appropriate; Vallée-Tourangeau \& March, 2019).

\section{Mental Arithmetic}

Mental arithmetic is another area where an interactivist approach can shed new light on cognitive performance. Mental arithmetic is a key skill; its development and expression are understood to rely on a person's working memory capacity (Raghubar, Barnes, \& Hecht, 2010) and moderated by mathematics anxiety (Ashcraft, 2002). Yet mental arithmetic can also be a situated skill enacted within a context or system configured through a person's actions that recruit and manipulate artefacts; these actions evince changes in the problem presentation that reduce working memory demands and shoulder computations. In other words, by paying attention to systemic working memory resources in modelling mathematical cognition or designing pedagogical interventions may offer a promising avenue for supporting learning and performance.

Research conducted on mental arithmetic specifically, and mathematical cognition generally, implicates working memory capacity as one of the key factors explaining performance (Logie, Gilhooly, \& Wynn, 1994; DeStefano \& Lefevre, 2004). Take the task of adding a long series of single-digit numbers, without recourse to pen or calculator, presented visually all at once in a random pattern. Calculating the correct answer requires temporary storage and executive skills: basic arithmetic facts and knowledge are retrieved to identify or calculate interim totals, which are rehearsed using the phonological loop (Baddeley, 2012); 
numbers are tagged as having been processed, others as those that need processing. Attentional resources are allocated to different regions of the visual array or switched to others to identify the next numbers to be added or backtrack and create more congenial subtotals. It appears to make sense to profile participants' working memory in order to predict their mental arithmetic ability and diagnose potential learning difficulties and pedagogical challenges.

Interactivity and Systemic Resources. Mental arithmetic is often naturally supported by a range of complementary actions, such as pointing, to guide attention and bind elements in a functional sequence (Kirsh, 1995; Carlson, Avraamides, Cary, \& Strasberg, 2007). Gesturing can also facilitate and speed up the processing information (Goldin-Meadow, Nusbaum, Kelly, \& Wagner, 2001). Imagine, again, adding a long series of single digit numbers, however, this time, the addition problem is presented with numbered tokens that can be manipulated to shape and re-shape the physical configuration of the problem. Mental arithmetic unfolds along a spatio-temporal itinerary produced by the reasoner's actions. These actions modify the problem and in so doing the problem is restructured, added numbers can be physically demarcated, no longer attracting attention, congenial interim sums (e.g., 8+7) are identified or physically created, they can be segregated to facilitate subsequent calculation; these changes in the problem presentation unveil new affordances of what to do next or as hitherto unnoticed complementary sub-totals (e.g., 9+6), that inter-lock to create easy-to-remember provisional totals (e.g., 30). Thus, interactivity can improve efficiency and reduce error (Vallée-Tourangeau, 2013). Changes in the problem's physical configuration may guide attention allocation decisions and shoulder some of the computational burden (Rumelhart, Smolensky, McClelland, \& Hinton, 1986).

The traditional cognitivist perspective from which most empirical and theoretical efforts on mental arithmetic proceeds, casts thinking in terms of an agent's cognitive 
capacities—such as working memory capacity—and dispositions—such as mathematics anxiety (e.g., Ashcraft \& Krause, 2007). Performance is measured in task environments that limits or prevents interacting with a problem. Participants' performance is then correlated with measures of capacity and dispositions, the same paradigm that guides research effort in problem solving reviewed earlier. Working memory capacity is a strong predictor of performance: the higher the working memory capacity, the lower the calculation error (Guthrie \&Vallée-Tourangeau, 2018). There is evidence that mathematics anxiety further constrains working memory resources through the explicit rumination of intrusive thoughts about unpleasant experiences involving maths teaching or testing; thus, maths anxiety positively correlates with calculation error, presumably because it further reduces processing capacity (Ashcraft, 2002).

A systemic perspective, in contrast, casts thinking as embodied and extended: it emerges from actions that modify the world and hence it is crucial to observe participants in interactive environments. The research proceeds on the basis of methodological interactivism (Bickhard, 2009). Interactivity couples a reasoner to her physical environment, configuring an extended cognitive system exploiting internal and external resource. On the one hand, interactivity reduces the demands on the reasoner's working memory resources, but on the other, the system's overall working memory resources are augmented: actions that modify a problem presentation may create new spatial arrangements that extend memory storage capacity, facilitate the allocation of attentional resources, and lighten the computational burden of the problem. It's not simply an extended storage story, however; more complex arithmetic strategies can be deployed in an interactive mental arithmetic task environment (Vallée-Tourangeau, 2013). Thus, a reasoner's working memory capacity and maths anxiety may predict mental arithmetic performance in a low interactivity reasoning environment but 
not in a high interactivity one. The theoretical and practical implications are important if this were the case.

We tested these ideas in an experiment that manipulated the type of interactivity as well as the internal working memory resources that a participant could deploy to complete addition problems (Vallée-Tourangeau, Sirota, \& Vallée-Tourangeau, 2016). Participants were presented long single-digit additions in either a low or high interactivity context. In the low interactivity one, the sums were presented on a side of A4 placed in front of the participants seated at a worktable. The numbers were randomly arrayed, and participants placed their hands palm down on the tabletop. They could not point to or touch the numbers; they could not use their fingers. In the high interactivity context, the same sums were presented with numbered tokens that participants could touch, move, manipulate as they saw fit in calculating the answer. Based on prior work, we expected that performance, as indexed by the absolute calculation error, would be better in the high interactivity context. We manipulated working memory resources through articulatory suppression: Thus, for some of the problems, participants were required to say 'the' continuously as they worked on a solution. This manipulation makes it much harder to verbally rehearse interim totals or maintain a verbal strategic narrative to guide problem solving, in other words it constrains and reduces the allocation of working memory resources to mental arithmetic. We expected performance to be substantially affected by articulatory suppression, with significantly greater calculation error with suppression than without. The experiment thus employed a 2 (interactivity: low, high) by 2 (articulatory suppression: with, without) design; both factors were manipulated within subjects, that is participants completed series of sums in all four experimental conditions (see Vallée-Tourangeau et al., 2016). We also measured participants' level of maths anxiety using a 25-item Mathematics Anxiety Scale-UK (Hunt, Clark-Carter \& Sheffield, 2011). These items ask participants to imagine how anxious they would feel in 
certain situations ( $1=$ "not at all" and $5=$ "very much"), such as "Working out how much your shopping bill comes to". We predicted that maths anxiety would moderate the impact of articulatory suppression on calculation error in the low interactivity condition but not in the high interactivity condition.

The results of the experiment supported our predictions. Thus, calculation error was significantly higher in the low interactivity than in the high interactivity condition.

Performance in both interactivity conditions was substantially affected by sewing up working memory resources through articulatory suppression. However, the performance decrement was significantly greater in the low interactivity condition, which suggests that the overall cognitive resources of the system created through interactivity could better absorbed the working memory depletion caused by articulatory suppression. The results of the moderation analysis involving maths anxiety and the increase in calculation error as a function of suppression are illustrated in Figure 3. The increase in calculation error was determined for each participant by taking the difference in calculation error with and without suppression; for most participants that difference was positive, that is greater calculation errors with than without suppression. These differences were regressed on the participants' level of mathematics anxiety (a moderation analysis for repeated measures designs as recommended in Judd, Kenny, \& McClelland, 2001). As the left panel illustrates, the correlation between level of maths anxiety and the impact of suppression was positive, and the regression model was significant. That is, as level of maths anxiety increased, articulatory suppression had a greater impact on calculation error. However, as the right panel illustrates, this was not the case in the high interactivity condition: levels of maths anxiety was not correlated with the increase in calculation error as a function of suppression. 

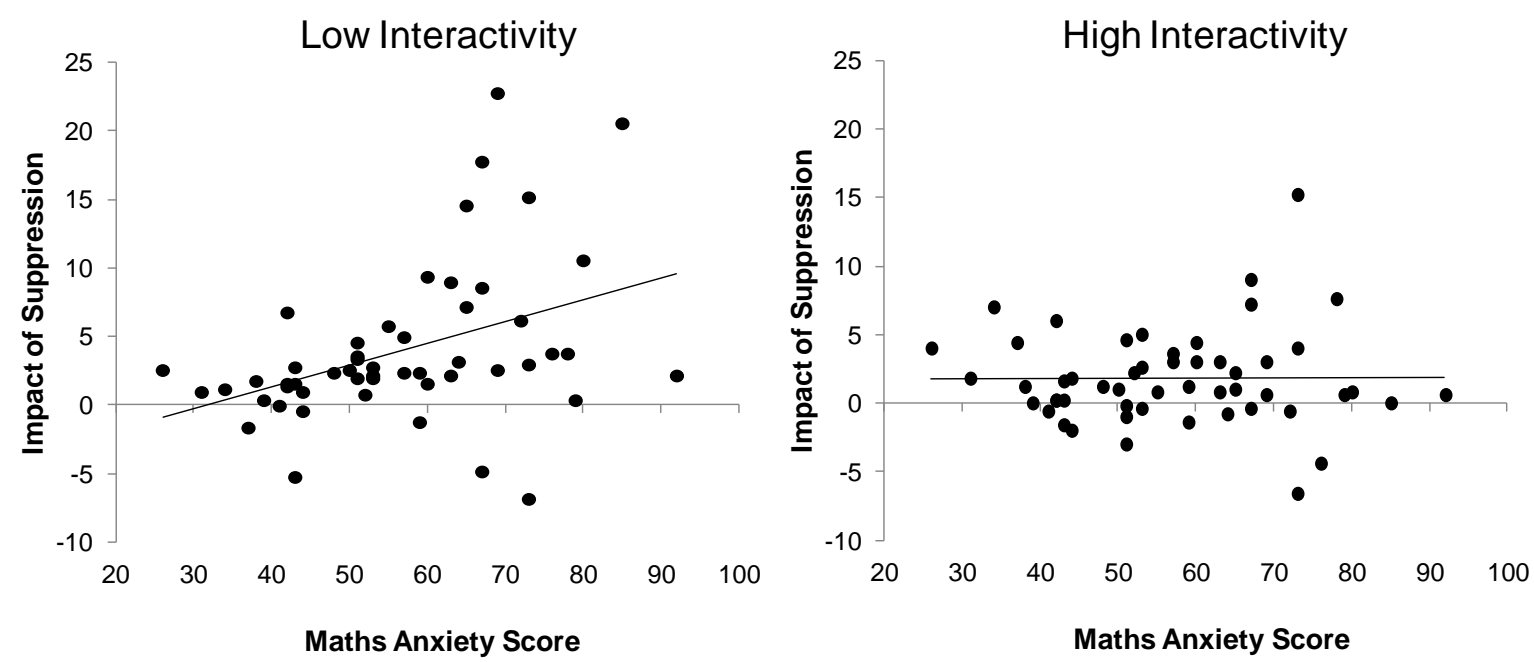

Figure 3. Correlation between level of mathematics anxiety and the increase in calculation error as a function of articulatory suppression in the low interactivity condition (left panel) and high interactivity condition (right panel). The descriptive and inferential statistics can be found in Vallée-Tourangeau et al. (2016).

The impact of articulatory suppression on performance clearly reflects the challenge posed by the dual task; the performance decrement was not surprising and fully expected. We used this dual task procedure to determine how well the augmented systemic resources assembled through the reasoner's action could absorb the additional processing burden: The impact of articulatory suppression on mental arithmetic was significantly weaker with interactivity. What is equally interesting is how maths anxiety moderated the impact of articulatory suppression on performance. When participants could not scaffold their thinking by interacting with a physical and malleable arithmetic problem, their level of maths anxiety exacerbated the debilitating impact of articulatory suppression. However, interactivity cancelled this ratchet effect, and participants' anxiety level did not enhance the performance decrements caused by suppression. This is not to say that internal resources do not contribute to performance in interactive tasks. Indeed, in mental arithmetic (e.g., Ross, ValléeTourangeau \& Van Herwegen, 2019) and Bayesian inference (Vallée-Tourangeau et al., 2015), and in other tasks, such as the word production task employed in Vallée-Tourangeau 
and Wrightman (2010) and Ross and Vallée-Tourangeau (2020), levels of numeracy and working memory (or verbal fluency in word production) moderate the impact of interactivity on performance: More numerate participants perform even better in interactive tasks environments. In Ross and Vallée-Tourangeau (2020), participants with high verbal fluency are better able to exploit the potential of an interactive word production task, moving individual letter tiles to produce words with greater speed and efficiency, unveiling more new words quickly.

There are several important implications. First, working memory is undoubtedly implicated in mental arithmetic, but a research methodology that ignores interactivity will continue to shed a distorting light on this link, be it causal or merely correlational. From a developmental and pedagogical perspective, the psychometric profiling of working memory capacity that ignores the cognitive ecosystems designed or improvised within which reasoning takes place will miss out on how arithmetic knowledge is retrieved and expressed. Second, interactivity mitigates the debilitating influence of maths anxiety on performance. This is particularly important in promoting a more positive experience with numerical tasks. Anxiety leads to the development of coping behaviour that reduces or eliminates exposure to stimuli that elicit the anxious reaction. The consequence of maths anxiety is self-reinforcing avoidance coping that further impedes the development of numeracy with important educational and occupational implications. Avoidance coping mechanisms can be defused by brokering encounters with feared stimuli in a manner that does not elicit anxiety, a form of extinction of learned responses (Mathews, 1978). Augmenting the systemic cognitive resources through interactivity promotes better performance and provides positive feedback which may work to reduce avoidance responses which may ultimately result in enhanced numeracy, with felicitous consequences. 


\section{Practical Implications}

The benefits of using physical objects to support cognitive activities has long been recognised. In the early nineteenth century, Friedrich Froebel, a German pedagogue, promoted the use of play with physical artefacts such as a ball and a set of wooden blocks to promote learning (Allen, 1988). Key figures in childhood development such as Piaget and Bruner have also long established that concrete materials can support children's problemsolving activity (Bruner, Olver, \& Greenfield, 1966; Piaget, 1953). Today, teachers still use "manipulatives" to support the development of mathematical thinking in younger children (Martignon, \& Krauss, 2009; see Ross et al., 2019). Far from being an element of the past, physical manipulatives may instead become more and more central to educational practices with the rise of ubiquitous computing (Weiser, 1991). Technology is no longer constrained to a desktop PC. Instead it is becoming part of everyday experiences, most commonly through the use of tangibles (e.g., electronic tablets). As tangibles develop the ability to interface with the physical world to creates digital events, novel form of interactive learning may emerge (e.g., Price, Rogers, Scaide, Stanton, \& Neale, 2003). In parallel, the field of "data physicalization" where data is encoded through geometry or material properties holds the promise to benefit cognition and learning (Jansen et al., 2015).

These technological developments, however, need to be better informed by a characterisation of how learning results from the cognitive transactions emerging as people evolve within their environment. When such environments are affordance-rich (i.e., replete with action possibilities), both agents and environment will co-evolve on a cognitive trajectory from which new ideas, insights, and learning emerge. As agents proceed along this spatiotemporal trajectory, they engage in cognitive transactions through a continuing process of reciprocal and emergent effects: the environment is modified by the actions of the thinking agent while the agent's internal cognitive resources are transformed by the changing 
environment (Turvey \& Shaw, 1999; Vallée-Tourangeau \& Vallée-Tourangeau, 2014). We need to better understand how those cognitive transactions are facilitated or hindered by individuals' characteristics and environmental features as well as their impact in space and time. There are two related conceptual stumbling blocks to overcome to make this possible: the first concerns current views on the developmental trajectory of cognition and the second concerns the ultimate locus of cognitive processing.

The Piagetian and Vygotskian models of cognitive development continue to guide much implicit beliefs about cognitive development (Goswami, 2002). Piaget proposed that children progress to three levels of knowing: knowing through action in infancy progresses towards knowing through representational thought in childhood and knowing through formal understanding in adolescence (Smith, 2002). Similarly, the ultimate cognitive achievement, according to Vygotsky's model is the transfer of processes originating in the social world into an inner "mental" world to shape the development of higher processes such as problemsolving (Gozwami, 2002). While some aspects of Piagetian and Vygtoskian views of development may have been debated, so called neo-Piagetian and neo-Vygotskian approaches inspired by information-processing models and artificial neural networks retain a key feature: they aim to model higher cognition as it ultimately evolves "inside the head" (e.g., Mareschal \& Shultz, 1999; Simon \& Klahr, 1995). This stance, we believe, is hindering progress for understanding how cognition emerges and evolves either in the moment or throughout the lifespan from dynamic organism-environment transactions. Neither children nor adults learners ultimately evolve to think solely "inside their head". They evolve to master the cognitive transactions they can harvest from their immediate digital and analogue environments.

According to the radical-embodiment thesis (e.g., Wilson \& Golonka, 2013) intelligent behaviour arises where the body meets environmental constraints and affordances. 
While we agree — and our work has consistently demonstrated — that we must forego the presupposition that higher cognition is the sole product of an agent's internal mental resources, we also believe that representational states are key to understand cognitive transactions. Thus, to move forward, we need to go beyond debates of the locus of cognition and focus instead on developing our understanding of how cognitive events emerge in time and space from the meshed transactions involving mental processes, behaviours, and environmental affordances.

\section{Concluding Remarks}

Methodological individualism undergirds an active, well-funded and long-standing programme of research in cognitive psychology. The research procedure employed in many reasoning and problem-solving experiment remains relatively narrow; participants' prosaic and indolent performance on these tasks may be a reflection of the research procedure rather than being representative of thinking outside the lab. In that respect the researchers themselves exhibit the very characteristics they deplore in their participants. Methodological individualism is at the heart of psychometric models of problem solving and learning; the concomitant neurocentrism plausibly drives efforts to map the neural correlates of various cognitive phenomena. This unremitting focus on the individual, and more specifically on what's inside her head, systematically biases the methods designed to offer a window onto these phenomena. The richly textured dynamic external environment, which its shifting set of action affordances, is reduced to psychometric exercises. In turn, neuroscience data can only be collected from immobile, and sometimes immobilized, participants.

In this paper we argue that interacting with a malleable problem presentation, and the consequent creation of a cognitive ecosystem transforms thinking. Methodologically, it becomes possible to provide a granular description of the dynamic configuration of this ecosystem from which new ideas emerge. Methodological interactivism offers the possibility 
of repaying the loan on intelligence, of explaining intelligent or creative problem solving in terms that do not themselves assume intelligence or creativity. As Ingold (2014, p. 124) puts it, "The wellsprings of creativity lie not inside people's head" Ingold (2014, p. 124) but rather through improvisation with the action possibilities of the material artefacts with which creativity is enacted. This, then, behoves us to map and describe how this is enacted. 


\section{References}

Allen, A. T. (1988). "Let us live with our children”: Kindergarten movements in Germany and the United States, 1840-1914. History of Education Quarterly, 28, 23-48. doi: $10.2307 / 368282$

Ashcraft, M. H. (2002). Math anxiety: Personal, educational and cognitive consequences. Current Directions in Psychological Research, 11, 181-185. doi: 10.1111/14678721.00196

Ashcraft, M. H., \& Krause, J. A. (2007). Working memory, math performance, and math anxiety. Psychonomic Bulletin \& Review, 14, 243-248. doi: 10.3758/BF03194059

Baddeley, A. (2012). Working memory: Theories, models and controversies. Annual Review of Psychology, 63, 1-29. doi: 10.1146/annurev-psych-120710-100422

Baumeister, R. F., Vohs, K. D., \& Funder, D. C. (2007). Psychology as the science of selfreports and finger movements. Perspectives on Psychological Science, 2, 396-403. doi: 10.1111/j.1745-6916.2007.00051.x

Bickhard, M. H. (2009). Interactivism: A manifesto. New Ideas in Psychology, 27, 85-95. doi:10.1016/j.newideapsych.2008.05.001

Bruner, J.S., Olver, R.R., \& Greenfield, P.M. (1966). Studies in cognitive growth. New York: John Wiley \& Sons.

Carlson, R. A., Avraamides, M. N., Cary, M., \& Strasberg, S. (2007). What to the hands externalize in simple arithmetic? Journal of Experimental Psychology: Learning, Memory, and Cognition, 33, 747-756. doi: 10.1037/0278-7393.33.4.747

Chuderski, A., \& Jastrzębski, J. (2018). Much ado about aha!: Insight problem solving is strongly related to working memory capacity and reasoning ability. Journal of Experimental Psychology: General, 147, 257-281. doi: 10.1037/xge000037 
Chuderski, A., Jastrzębski, J., \& Kucwaj, H. (2020). How physical interaction with insight problems affects solution rates, hint use, and cognitive load. British Journal of Psychology. doi:10.1111/bjop.12442

Clark, A. (2010). Material surrogacy and the supernatural: Reflections on the role of artefacts in 'off-line' cognition. In L. Malafouris and C. Renfrew (Eds.), The cognitive life to things (pp. 23-28). Cambridge: McDonald Institute for Archaeological Research.

Copeland, S. M. (2018). "Fleming leapt on the unusual like a weasel on a vole": Challenging the paradigms of discovery in science. Perspectives on Science, 26, 694-721. doi:10.1162/posc_a_00294

Daneman, M., \& Carpenter, P. A. (1980). Individual differences in working memory and reading. Journal of Verbal Learning and Verbal Behavior, 19, 450-466. doi:10.1016/S0022-5371(80)90312-6

Davidson, J. (1995). The suddenness of insight. In R. J. Sternberg and J. E. Davidson (Eds.), The nature of insight (pp. 125-155). Cambridge MA: The MIT Press.

DeStefano, D., \& LeFevre, J. (2004). The role of working memory in mental arithmetic. European Journal of Cognitive Psychology, 16, 353-386. doi:10.1080/09541440244000328

Engle, R. W., Tuholski, S. W., Laughlin, J. E., \& Conway, A. R. A. (1999). Working memory, short-term memory, and general fluid intelligence: A latent-variable approach. Journal of Experimental Psychology: General, 128, 309-331. doi:10.1037/00963445.128.3.309

Fleck, J. I., \& Weisberg, R. W. (2013). Insight versus analysis: Evidence for diverse methods in problem solving. Journal of Cognitive Psychology 25, 436-463. doi: 10.1080/20445911.2013.779248 
Frederick, S. (2005). Cognitive reflection and decision making. Journal of Economic Perspectives, 19, 25-42. doi:10.1257/089533005775196732

Fiore, S. M., \& Wiltshire, T. J. (2016) Technology as teammate: Examining the role of external cognition in support of team cognitive processes. Frontiers in Psychology, 7, 1531. doi:10.3389/fpsyg.2016.01531

Gilhooly, K. J., \& Fioratou, E. (2009). Executive functions in insight versus non-insight problem solving: An individual differences approach. Thinking and Reasoning, 15, 355-376. doi:10.1080/13546780903178615

Gilhooly, K. J., Georgiou, G., \& Devery, U. (2013). Incubation and creativity: Do something different. Thinking \& Reasoning, 19, 137-149. doi:10.1080/13546783.2012.749812

Glăveanu, V., Lubart, T., Bonnardel, N., Botella, M., de Biasi, P.-M., et al. (2013). Creativity as action: Findings from five creative domains. Frontiers in Psychology, 4, 176. doi:10.3389/fpsyg.2013.00176

Goldin-Meadow, S., Nusbaum, H., Kelly, S. D., \& Wagner, S. (2001). Explaining math: Gesturing lightens the load. Psychological Science, 12, 516-522. doi: 10.1111/14679280.00395

Goswami, U. (2002). Models of cognitive development. In U. Goswami (Ed.), Blackwell handbook of cognitive development (pp. 509-514). Malden, MA: Blackwell Publishers.

Guthrie, L. G., \& Vallée-Tourangeau, F. (2018). Numbers in action: Individual differences and interactivity in mental arithmetic. Cognitive Processing, 19, 317-326. doi:10.1007/s10339-018-0856-7

Hunt, T. E., Clark-Carter, D., \& Sheffield, D. (2011) The development and part validation of a UK scale for mathematics anxiety. Journal of Psychoeducational Assessment, 29, 455-466. doi: 10.1177/0734282910392892

Hutchins, E. (1995). Cognition in the wild. Cambridge, MA: MIT Press. 
Hutchins, E. (2010). Cognitive ecology. Topics in Cognitive Science, 2, 705-715. doi: 10.1111/j.1756-8765.2010.01089.x

Ingold, T. (2014). The creativity of undergoing. Pragmatics \& Cognition, 22, 124-139. doi:10.1075/pc.22.1.07ing

Jansen, Y., Dragicevic, P., Isenberg, P., Alexander, J., Karnik, A., Kildal, J., Subramanian, S., \& Hornbæk, K. (2015). Opportunities and challenges for data physicalization (pp. 3227-3236). ACM Press. doi:10.1145/2702123.2702180

Judd, C. M., Kenny, D. A., \& McClelland, G. H. (2001). Estimating and testing mediation and moderation in within-subject designs. Psychological Methods, 6, 115-134. doi: 10.1037/1082-989X.6.2.115

Kirsh, D. (1995). Complementary strategies: Why we use our hands when we think. In J. M. Moore \& J. L. Lehman (Eds.), Proceedings of the Seventeenth Annual Conference of the Cognitive Science Society (pp. 212-217). Mahwah, NJ: Lawrence Erlbaum Associates, Publishers.

Kirsh, D. (2009). Problem solving and situated cognition. In, P. Robbins \& M. Aydede (Eds.), The Cambridge handbook of situated cognition (pp. 264-306). Cambridge: Cambridge University Press.

Kirsh, D. (2010). Thinking with external representations. AI \& Society, 25, 441-454. doi:10.1007/s00146-010-0272-8

Kirsh, D. \& Maglio, P. (1994). On distinguishing epistemic from pragmatic action. Cognitive Science, 18, 513-549. doi:10.1207/s15516709cog1804_1

Kounios, J., \& Beeman, M. (2014). The cognitive neuroscience of insight. Annual Review of Psychology, 65, 71-93. doi:10.1146/annurev-psych-010213-115154

Latour, B. (1999). Pandora's hope. Harvard University Press. 
Latour, B., \& Woolgar, S. (1986). Laboratory life. The construction of scientific facts (second edition). Princeton University Press.

Lave, J. (1988). Cognition in practice: Mind, mathematics and culture in everyday life. New York: Cambridge University Press.

Logie, R. H., Gilhooly, K. J., \& Wynn, V. (1994). Counting on working memory in arithmetic problem solving. Memory \& Cognition, 22, 395-410. doi: 10.3758/BF03200866

Malabou, C. (2019). Morphing intelligence: From IQ measurement to artificial brains. Columbia University Press.

Malafouris, L. (2014). Creative thinging: The feeling of and for clay. Pragmatics \& Cognition, 22, 140-158. doi:10.1075/pc.22.1.08mal

Malafouris, L. (2015). Metaplasticity and the primacy of material engagement. Time and Mind: The Journal of Archaeology, Consciousness and Culture, 8, 351-371. doi:10.1080/1751696X.2015.1111564

March, P. L. (2019). Playing with clay and the uncertainty of agency. A Material Engagement Theory perspective. Phenomenology and the Cognitive Sciences, 18, $133-$ 151. doi:10.1007/s11097-017-9552-9

Mareschal, D., \& Shultz, T. R. (1999). Development of children's seriation: A connectionist approach. Connection Science, 11, 149-186. doi:10.1080/095400999116322

Martignon, L., \& Krauss, S. (2009). Hands-on activities for fourth graders: A tool box for decision-making and reckoning with risk. International Electronic Journal of Mathematics Education, 4, 227-258.

Mathews, A. (1978). Fear-reduction research and clinical phobias. Psychological Bulletin, 85, 390-404. doi:10.1037/0033-2909.85.2.390

Piaget, J. (1953). How children form mathematical concepts. Scientific American, 189, 74-79. 
Pitts-Taylor, V. (2010). The plastic brain: Neoliberalism and the neuronal self. Health, 14, 635-652. doi: 10.1177/1363459309360796.

Price, S., Rogers, Y., Scaife, M., Stanton, D., \& Neale, H. (2003). Using “tangibles” to promote novel forms of playful learning. Interacting with Computers, 15(2), 169-185. doi:10.1016/S0953-5438(03)00006-7

Raghubar, K. P., Barnes, M. A., \& Hecht, S. A. (2010). Working memory and mathematics: A review of developmental, individual difference, and cognitive approaches. Learning and Individual Differences, 20, 110-122. doi:10.1016/j.lindif.2009.10.005

Ross, W., \& Vallée-Tourangeau, F. (2020). Catch that word: Interactivity, serendipity and verbal fluency in a word production task. Psychological Research. doi: 10.1007/s00426-019-01279-y

Ross, W., Vallée-Tourangeau, F., \& Van Herwegen, J. (2019). Mental arithmetic and interactivity: The effect of manipulating external number representations on older children's mental arithmetic success. International Journal of Science and Mathematics Education. doi: 0.1007/s10763-019-09978-z

Rumelhart, D. E., Smolensky, P., McClelland, J. L., \& Hinton, G. E. (1986). Schemata and sequential thought processes in PDP models. In: J. L. McClelland, D. E. Rumelhart, \& the PDP Research Group (Eds), Parallel distributed processing: Explorations in the microstructure of cognition, Vol. 2 (pp. 7-57). Cambridge MA: MIT Press.

Simon, T., \& Klahr, D. (1995). A computational theory of children's learning about number conservation. In T. Simon \& G. S. Halford (Eds.), Developing cognitive competence: New approaches to process modelling (pp. 315-353). Hillsdale, NJ: Erlbaum.

Smith, L. (2002). Piaget's model. In Goswami (Ed.), Blackwell handbook of cognitive development (pp. 515-537). Malden, MA: Blackwell Publishers. 
Stanovich, K. E., \& West, R. F. (1998). Individual differences in rational thought. Journal of Experimental Psychology: General, 127, 161-188. doi:10.1037/0096-3445.127.2.161

Toplak, M. E., West, R. F., \& Stanovich, K. E. (2011). The Cognitive Reflection Test as a predictor of performance on heuristics-and-biases tasks. Memory \& Cognition, 39, 1275-1289. doi:10.3758/s13421-011-0104-1

Turvey, M. T., \& Shaw, R. E. (1999). Ecological foundations of cognition. I: Symmetry and specificity of animal-environment systems. Journal of Consciousness Studies, 6, 95110.

Vallée-Tourangeau, F. (2013). Interactivity, efficiency, and individual differences in mental arithmetic. Experimental Psychology, 60, 302-311. doi:10.1027/1618-3169/a000200

Vallée-Tourangeau, F. (Ed.) (2018). Insight: On the origins of new ideas. London: Routledge.

Vallée-Tourangeau, F., \& March, P. L. (2019). Insight out: Making creativity visible. Journal of Creative Behavior. doi: 10.1002/jocb.409

Vallée-Tourangeau, F., Sirota, M., \& Vallée-Tourangeau, G. (2016). Interactivity mitigates the impact of working memory depletion on mental arithmetic performance. Cognitive Research: Principles and Implications, 1, 26. doi: 10.1186/s41235-016-0027-2

Vallée-Tourangeau, F., Steffensen, S. V., Vallée-Tourangeau, G., \& Sirota, M. (2016). Insight with hands and things. Acta Psychologica, 170, 195-205. doi:10.1016/j.actpsy.2016.08.006

Vallée-Tourangeau, F., \& Vallée-Tourangeau, G. (2014). Diagrams, jars and matchsticks: A systemicist's toolkit. Pragmatics \& Cognition, 22, 187-205. doi:10.1075/pc.22.2.02val

Vallée-Tourangeau, F., \& Vallée-Tourangeau, G. (2020). Insight. In V.P. Glãveanu (Ed.), Palgrave Encyclopedia of the Possible. doi: 10.1007/978-3-319-98390-5_35-1 
Vallée-Tourangeau, G., \& Vallée-Tourangeau, F. (2014). The spatio-temporal dynamics of systemic thinking. Cybernetics \& Human Knowing, 21, 113-127.

Vallée-Tourangeau, G., Abadie, M., \& Vallée-Tourangeau, F. (2015). Interactivity fosters Bayesian reasoning without instruction. Journal of Experimental Psychology: General, 144, 581-603. doi: 10.1037/a0039161

Wason, P. C. (1966). Reasoning. In B. M. Foss (Ed.), New horizons in psychology I. Harmondsworth: Penguin.

Wason, P.C. (1960). On the failure to eliminate hypotheses in a conceptual task. Quarterly Journal of Experimental Psychology, 12, 129-140. doi:10.1080/17470216008416717

Weisberg, R. W. (1993). Creativity: Beyond the myth of genius. W. H. Freeman.

Weisberg, R. W. (1995). Prolegomena to theories of insight in problem solving: A taxonomy of problems. In R. J. Sternberg \& J. E. Davidson (Eds.), The nature of insight (pp. 157196). Cambridge MA: MIT Press.

Weisberg, R. W. (2006). Creativity: Understanding innovation in problem solving, science, invention, and the arts. Wiley.

Weisberg, R. W. (2018). Insight, problem solving, and creativity: An integration of findings. In F. Vallée-Tourangeau (Ed.), Insight: On the origins of new ideas (pp. 191-215). London: Routledge.

Weiser, M. (1991). The computer for the 21st century. Scientific American, 265, 94-104. doi:10.1038/scientificamerican0991-94

Wilson, A. D., \& Golonka, S. (2013). Embodied cognition is not what you think it is. Frontiers in Psychology, 4, 58. doi:10.3389/fpsyg.2013.00058

Zabelina, D. L., Condon, D., \& Beeman, M. (2014). Do dimensional psychopathology measures relate to creative achievement or divergent thinking? Frontiers in Psychology, 5, 1029. doi: 10.3389/fpsyg.2014.01029 